\title{
Efficient 2D Filtering for Cone-beam VOI Reconstruction
}

\author{
Yan Xia, Student Member, IEEE, Andreas Maier, Frank Dennerlein, Member, IEEE, Hannes G. Hofmann, \\ and Joachim Hornegger, Member, IEEE
}

\begin{abstract}
In some clinical applications, e.g., examination of deployed stents or coils during the intervention, only a small portion of the patient may be of diagnostic interest. For the sake of dose reduction to the patient, it is practicable to deploy a collimator to block radiation dose outside volume of interest (VOI). The resulting truncation, however, particularly in lateral direction, poses a challenge to the conventional reconstruction methods. The Approximated Truncation Robust Algorithm for Computed Tomography (ATRACT) is able to reconstruct images without the use of any explicit extrapolation schemes, even for highly truncated data. It is based on a decomposition of the standard ramp-filter into a local and a non-local filtering step, where the local step coincides with the two-dimensional (2D) Laplace operator and the non-local step is a 2D Radon-based filtering. In a practical implementation, the Radon-based filtering is not computationally efficient. In this paper, we present an improvement of the original ATRACT algorithm. The 2D Radonbased filtering step in the original algorithm is replaced by an analytical $2 \mathrm{D}$ convolution, resulting in a significant improvement in computational performance while retaining the image quality benefits of the VOI algorithm.
\end{abstract}

Index Terms-Image reconstruction, volume of interest, truncation correction, dose reduction

\section{INTRODUCTION}

$\mathbf{O}$ $\mathrm{NE}$ of the issues in radiology today is how to reduce the dose without compromising image quality. In some clinical workflows, e.g. examination of deployed stents, cochlear implants, and needle biopsies, only a small portion of the patient may be of diagnostic interest. For the sake of dose reduction to the patient, it is practicable to deploy a collimator to block radiation dose outside the VOI during the image acquisition, as illustrated in Fig. 1. However, the resulting truncation by the collimation, particularly in lateral direction, introduces a non-linear artifact in the reconstructions. This non-linear artifact (often referred to as the cupping artifact or generally the truncation artifact) typically manifests as a bright

Manuscript received May 7, 2012. This work was supported by Siemens AG, Healthcare Sector and of the Erlangen Graduate School in Advanced Optical Technologies (SAOT) by the German Research Foundation (DFG) in the framework of the German excellence initiative. The concepts and information presented in this paper are based on research and are not commercially available.

Y. Xia, H. G. Hofmann, and J. Hornegger are with the Pattern Recognition Lab, Friedrich-Alexander-University Erlangen-Nuremberg, 91058 Erlangen, Germany. Y. Xia and J. Hornegger are also with the Erlangen Graduate School in Advanced Optical Technologies (SAOT), Friedrich-Alexander-University Erlangen-Nuremberg, 91052 Erlangen, Germany (e-mail: yan.xia@cs.fau.de; hannes.hofmann@cs.fau.de; joachim.hornegger@cs.fau.de).

A. Maier and F. Dennerlein are with Siemens AG, Healthcare Sector, 91301 Forchheim, Germany (e-mail: ammaier@siemens.com; frank.dennerlein@siemens.com).

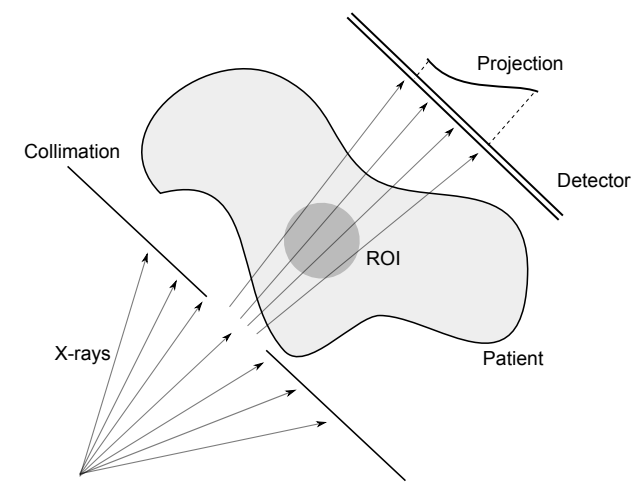

Fig. 1. Setup for VOI imaging.

ring near the border of the VOI and considerably contaminates the image quality in the reconstructions. Therefore, it is of practical significance to develop algorithms for VOI imaging that are accurately comparable to reconstructions from the non-truncated projections.

So far various correction methods have been proposed to overcome the effect of truncation artifact. Approaches in Noo et al. [1], Pan et al. [2], Defrise et al. [3], Ye et. al [4], [5], Kudo et al. [6], Chityala et al. [7], Koloditz et al. [8], [9] can provide exact or high accurate reconstruction for imaging. But they require either prior information of the object, or additional radiation dose, scans of patient shape and outline. Some are even computationally intensive and complicated.

Other truncation correction methods are based on explicit extrapolation of missing data using various strategies. Examples of this type are symmetric mirroring of projection images (Ohnesorge et al. [10]), approximation of the patient as a water cylinder (Hsieh et al. [11]), square root extrapolation (Sourbelle et al. [12]), water ellipse extrapolation (Maltz et al. [13]), or optimization-based extrapolation (Maier et al. [14]). However, these methods are based on heuristic extrapolation, which may not always accurately approximate the objects outside the VOI.

Recently, a novel method for VOI reconstruction of highly truncated projection data with neither the use of prior knowledge nor any explicit extrapolation has been published by Dennerlein [15]. It is called the Approximated Truncation Robust Algorithm for Computed Tomography (ATRACT) and is based on a decomposition of the standard ramp filter within FDK (Feldkamp, Davis, and Kress algorithm [16]) into a local and a non-local filtering step, where the local step is a $2 \mathrm{D}$ Laplace operator and the non-local step is a 2D Radon-based 
filtering. The non-local filtering consisting of Radon transform and inverse Radon transform, is computationally demanding. This limits the deployment of the ATRACT algorithm in clinical use. It is our goal to simplify the filtering procedure in the original ATRACT algorithm without compromising robustness of truncation correction.

\section{MATERials AND Methods}

\section{A. FDK Algorithm}

The FDK algorithm [16] is specifically dedicated to the circular cone-beam trajectory. It is a simple but effective extension of a $2 \mathrm{D}$ fan-beam FBP algorithm to 3D conebeam data. Fig. 2 shows the associated notations in the conebeam imaging geometry. The mathematical expression of 2D projection data $g(\lambda, u, v)$ can be written as follows:

$$
g(\lambda, u, v)=\int_{0}^{\infty} f(\mathbf{a}(\lambda)+t \boldsymbol{\alpha}(\lambda, u, v)) d t .
$$

The standard FDK algorithm consists of the following three steps:

- Step 1: Cosine- and Parker-like weighting of projection data to obtain pre-scaled projection data $g_{1}(\lambda, u, v)$

$$
g_{1}(\lambda, u, v)=\frac{D m(\lambda, u)}{\sqrt{D^{2}+u^{2}+v^{2}}} g(\lambda, u, v),
$$

where $m$ denotes a Parker weight which is constantly 0.5 for a full circular scan but has to be determined to approximate the data redundancy for a short scan.

- Step 2: 1D row-wise ramp filtering to obtain filtered projection data $g_{F}(\lambda, u, v)$ :

$$
g_{F}(\lambda, u, v)=\int_{-\infty}^{\infty} h_{R}\left(u-u^{\prime}\right) g_{1}\left(\lambda, u^{\prime}, v\right) d u^{\prime},
$$

where $h_{R}(u)$ is the ramp filter kernel in the spatial domain.

- Step 3: 3D cone-beam backprojection with a weighting function of object-focal point distance to get the estimated object function $f^{(F D K)}(\mathbf{x})$ :

$$
f^{(F D K)}(\mathbf{x})=\int_{\lambda_{1}}^{\lambda_{2}} \frac{R D}{\left[R-\mathbf{x} \cdot \mathbf{e}_{w}(\lambda)\right]^{2}} g_{F}(\lambda, u, v) d \lambda,
$$

where $\mathbf{x}=(x, y, z)$ and $\left[\lambda_{1}, \lambda_{2}\right]$ is the short scan angular range.

\section{B. Original ATRACT Algorithm}

Intuitively, the idea behind ATRACT is to decompose the ramp filter with the FDK algorithm into two successive 2D filtering steps [15]. The first step is a 2D Laplace operation. It is a local operation and can be computed efficiently in the spatial domain. The second step is a 2D Radon-based filtering. It is a non-local filtering and consists of the two following parts:

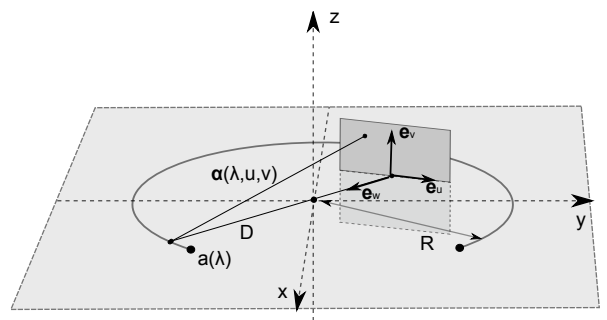

Fig. 2. Cone-beam geometry and associated notation: The curve $\mathbf{a}(\lambda)=$ $(R \cos \lambda, R \sin \lambda, 0)$ describes the trajectory of the $\mathrm{X}$-ray source, with $\mathrm{R}$ being the scan radius and $\lambda$ being the rotation angle. The planar detector is parallel to the unit vectors $\mathbf{e}_{\mathbf{u}}(\lambda)$ and $\mathbf{e}_{\mathbf{v}}(\lambda)$ and at distance D from the source. $\mathbf{e}_{\mathbf{w}}(\lambda)$ is the detector normal. We use the function $g(\lambda, u, v)$ to describe the projection data at the point $(\mathrm{u}, \mathrm{v})$ acquired at angle $\lambda$.

$$
\begin{aligned}
& g_{2}(\lambda, \theta, s)=\iint_{\Omega} g_{1}(\lambda, u, v) \delta\left(\mathbf{u}\left(\begin{array}{c}
\cos \theta \\
\sin \theta
\end{array}\right)-s\right) d \mathbf{u} \\
& g_{F}^{(A T R A C T)}(\lambda, u, v)=\frac{1}{4 \pi^{2}} \frac{R}{D} \int_{0}^{\pi}|\cos \theta| g_{2}\left(\lambda, \theta, s^{*}\right) d \theta
\end{aligned}
$$

where $\Omega$ denotes the shadow of the object on the detector plane, $\theta$ denotes the angle between the line normal vector and the $u$-axis, $|s|$ is the distance between the line and the origin $(0,0), \delta$ is the Dirac delta function, $\mathbf{u}=(u, v)$ and $s^{*}=u \cos \theta+v \sin \theta$

\section{Derivation of Analytical ATRACT}

The non-local filtering in the ATRACT algorithm consisting of Eqns. (5) and (6) is computationally expensive. The concept of the $2 \mathrm{D}$ convolution was suggested in [17], the corresponding kernel was estimated numerically. In the practical implementation, (5) and (6) have to be executed once for determining the 2D filtering kernel. Moreover, Eqns. (5) and (6) require many interpolations. Therefore, a loss in spatial resolution of the reconstruction is unavoidable.

We now derive the analytical convolution formula that replaces the 2D Radon-based filter in the original ATRACT algorithm. The new method has potential to increase computational performance and reduces the computational time for practical use.

Eqn. (5) can be rewritten as follows:

$g_{2}(\lambda, \theta, s)=\int_{-\infty}^{\infty} g_{1}(\lambda, s \cos \theta-t \sin \theta, s \sin \theta+t \cos \theta) d t$.

Inserting (7) into (6) yields

$$
\begin{aligned}
& g_{F}^{(\operatorname{ATRACT})}(\lambda, u, v)=\frac{1}{4 \pi^{2}} \frac{R}{D} \int_{0}^{\pi}|\cos \theta| \\
& \int_{-\infty}^{\infty} g_{1}\left(\lambda, s^{*} \cos \theta-t \sin \theta, s^{*} \sin \theta+t \cos \theta\right) d t d \theta .
\end{aligned}
$$




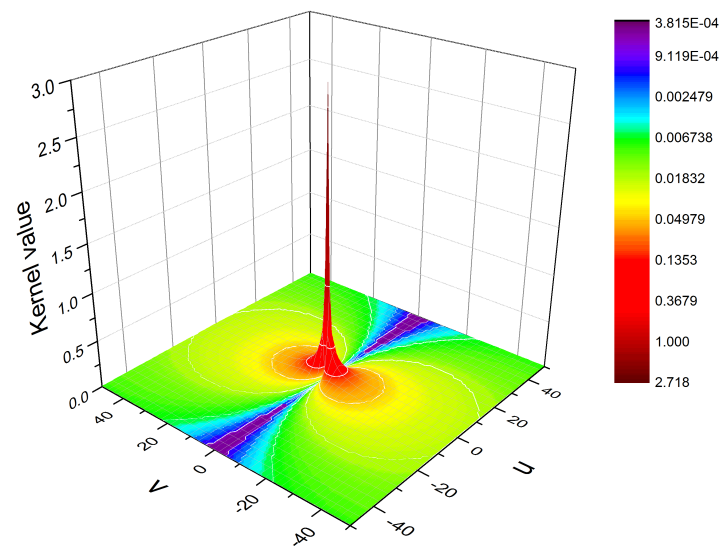

Fig. 3. 3D plot of the analytical kernel. The center value is estimated as 3 .

Then, we substitute variables $(t, \theta)$ by $\left(u^{\prime}, v^{\prime}\right)$ with $u^{\prime}=t \sin \theta$ and $v^{\prime}=-t \cos \theta$ and compute the corresponding Jacobian as follows:

$$
\begin{aligned}
d u^{\prime} & =\frac{\partial u^{\prime}}{\partial t} d t+\frac{\partial u^{\prime}}{\partial \theta} d \theta \quad \text { and } \\
d v^{\prime} & =\frac{\partial v^{\prime}}{\partial t} d t+\frac{\partial v^{\prime}}{\partial \theta} d \theta .
\end{aligned}
$$

Eqns. (9) and (10) can be rewritten into matrix form and solved for $\left[\begin{array}{l}d t \\ d \theta\end{array}\right]$

$$
\left[\begin{array}{l}
d t \\
d \theta
\end{array}\right]=\mathbf{J}^{-1}\left[\begin{array}{l}
d u^{\prime} \\
d v^{\prime}
\end{array}\right],
$$

where $\mathbf{J}^{-1}$ denotes the inverse Jacobian. It is calculated as

$$
\mathbf{J}^{-1}=\left[\begin{array}{cc}
\sin \theta & -\cos \theta \\
\frac{\cos \theta}{t} & \frac{\sin \theta}{t}
\end{array}\right] .
$$

Since we set $u^{\prime}=t \sin \theta$ and $v^{\prime}=-t \cos \theta$, we can also obtain

$$
\begin{aligned}
|t| & =\sqrt{u^{\prime 2}+v^{\prime 2}} \quad \text { and } \\
|\cos \theta| & =\frac{\left|v^{\prime}\right|}{\sqrt{u^{\prime 2}+v^{\prime 2}}} .
\end{aligned}
$$

Now, inserting (11), (12), (13) and (14) into (8), we obtain the 2D convolution formulation

$$
\begin{array}{r}
g_{F}^{(A T R A C T)}(\lambda, u, v)=\int_{u_{1}}^{u_{2}} \int_{v_{1}}^{v_{2}} g_{1}\left(\lambda, u-u^{\prime}, v-v^{\prime}\right) \\
h_{2 D}\left(u^{\prime}, v^{\prime}\right) d u^{\prime} d v^{\prime},
\end{array}
$$

where $u=s^{*} \cos \theta$ and $v=s^{*} \sin \theta$. The formula of $h_{2 D}\left(u^{\prime}, v^{\prime}\right)$ is determined as follows:

$$
h_{2 D}\left(u^{\prime}, v^{\prime}\right)=\frac{1}{4 \pi^{2}} \frac{R}{D}|\cos \theta|\left|\operatorname{det}\left(\mathbf{J}^{-1}\right)\right|=\frac{1}{4 \pi^{2}} \frac{R}{D} \frac{\left|v^{\prime}\right|}{u^{\prime 2}+v_{(16)}^{\prime 2}} \text {. }
$$

The plot of the 2D analytical kernel is shown in Fig. 3. Note that reconstruction resolution can be controlled for the newly proposed algorithm by applying a Gaussian distribution function on (16). Throughout this paper, the ATRACT with the 2D analytical convolution will be referred to as 2D ATRACT.

\section{Experiment Setup}

The proposed algorithms were validated and evaluated by the following data sets in terms of computational efficiency and robustness of correction quality.

1) Measurement of Computational Performance: To analyze the computational efficiency of the new algorithm, an open reconstruction benchmark (RabbitCT [18]) was used. It is an open-source testing benchmark for comparison of the reconstruction performance using a specific high resolution C-arm CT (Artis Zee, Siemens AG) data set of a rabbit. This data set contains 496 projection images acquired on a $200^{\circ}$ short-scan circular trajectory. Each of the projection images is 1240 pixels in width and 960 pixels in height at an isotropic resolution of $0.308 \mathrm{~mm} /$ pixel.

The evaluation of the computational performance in the proposed algorithm and the original one was carried out by measuring the execution time spent on processing of 496 full FOV projections of RabbitCT. Additionally, we implemented the standard ramp filter in CPU and compared it to our new methods. The corresponding GPU versions were also implemented using CUDA (CUDA 4.0). Note that since the Radon-based filtering operation in the original ATRACT needs interpolations, the resolution was matched by choosing the appropriate number of angular samples $\theta$ and radial samples $s$ in Eqns. (5) and (6).

2) Measurement of Correction Quality: We used two clinical examples in presence of different types of truncation, to quantify the robustness of the proposed algorithms for practical application. All data sets were acquired on a Carm CT system (Artis Zee, Siemens AG) from St. Lukes Episcopal Hospital (Houston, TX, USA). All scans containing 496 projection images $(960 \times 1240)$ with the resolution of $0.308 \mathrm{~mm} /$ pixel $(2 \times 2$ binning mode $)$ were acquired on a $200^{\circ}$ short-scan circular trajectory.

Fig. 4 exemplarily shows two clinical cases. Case I (first row) is a medium truncation case. The FOV of truncated projection is about $29 \%$ of corresponding full FOV scan. The dose reduction in the VOI scan is also reduced by $70 \%$ with respect to the full FOV scan. Case II (second row) was used to test the impact of severe truncation on the proposed algorithm. The FOV of truncated projection is only $15 \%$ of full FOV scan. Here the dose was not measured since we only deployed a virtually collimation. Also note that the truncation is offcentered.

All clinical data were reconstructed onto a Cartesian grid $(512 \times 512 \times 350)$ with sampling spacing $\triangle x=\triangle y=$ $\triangle z=0.4 \mathrm{~mm}$. The standard FDK reconstruction of full FOV scan was used as the gold standard in each case. The VOI scans were reconstructed by the proposed correction. We also investigated the FDK method from the VOI scans without the use of any correction method, and compare it to our new method. For the evaluation, resolution was matched by computing modulation transfer function (MTF) of the 

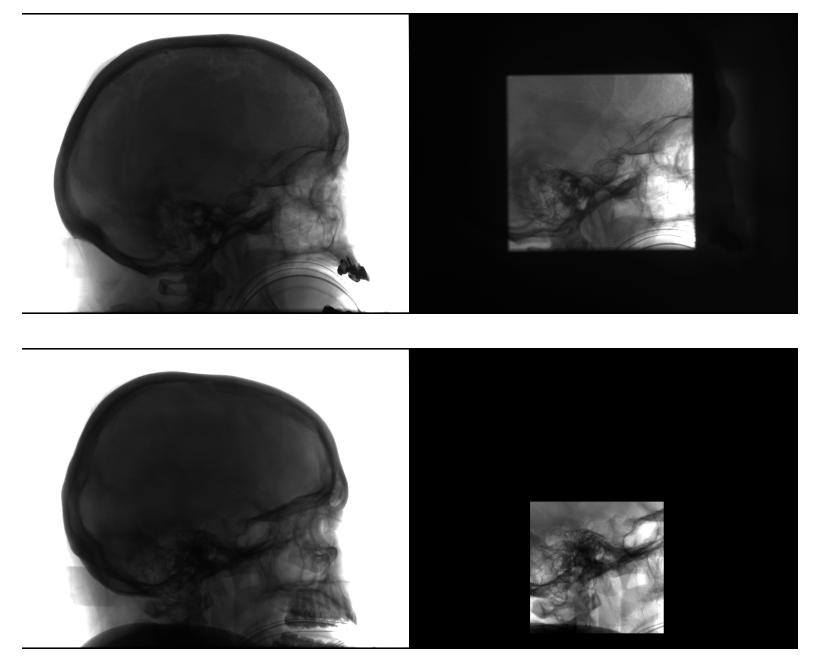

Fig. 4. Projection images of the clinical data sets. Left column: the full FOV scan, right column: the corresponding VOI scan.

new ATRACT reconstructions and FDK reconstruction with a Shepp-Logan filter using a bead phantom.

\section{RESULTS}

\section{A. Computational Performance}

The speed-up factors with respect to the original ATRACT for each implementation are shown in Fig. (5). The blue bars represent $\mathrm{CPU}$ implementations and green bars represent the corresponding CUDA implementations. As expected, the 2D analytical ATRACT is much more efficient. It speeds the processing up by 45 times of the original one since it avoids the Radon-based filtering by using a 2D Cartesian convolution kernel in the projection coordinates.

Intuitively, further improvement in computational performance is gained by using a high-parallel compute unit - the GPU. A NVIDIA Quadro FX 5800 was used for the GPU implementations, in CUDA 4.0. It provides a tremendous computational horsepower due to its 240 CUDA-programmable parallel cores with $4 \mathrm{~GB}$ of memory space. The CUDA version of the Original ATRACT speeds 60 times up to its CPU version. This improvement can be explained by the additional benefit from the utilization of texture memory which can be faster accessible and is able to implicitly handle linear and bilinear interpolations. In the CUDA versions of 2D ATRACT, FFT-based convolutions are provided in the form of an API, which achieves a speed-up factor of 860 with respect to the original one.

\section{B. Truncation correction}

The reconstruction results of clinical case I are represented Fig. 6. As expected, the typical bright ring artifact arises when no truncation correction is used for FDK. This degraded image limits its actual diagnostic value. As opposed to the FDK method, the reconstruction of high accuracy is maintained by the new algorithm. No bright ring artifact is observed in the FOV, which implies that high frequency artifact is essentially suppressed by the new method. At the same time,

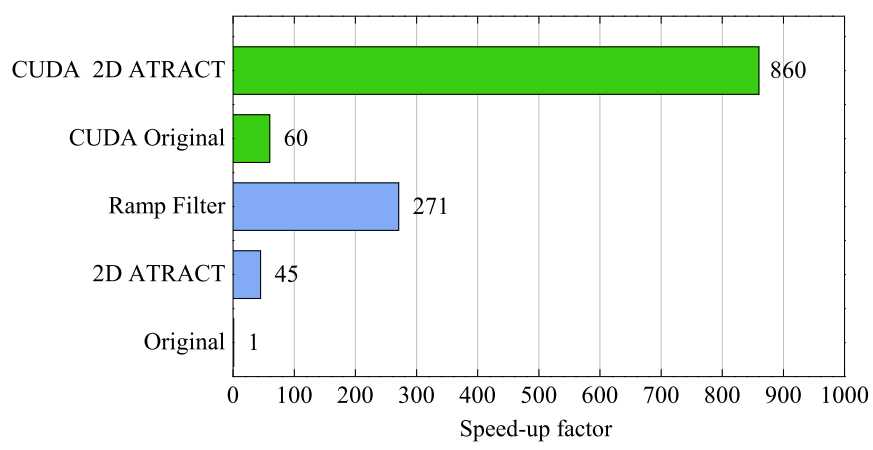

Fig. 5. Speed-up factor comparison of the different versions of ATRACT and the ramp filter by processing 496 projection images of RabbitCT. "Original" stands for the direct implementation of Eqns. (5) and (6), "2D ATRACT" is the 2D FFT-based implementation of Eqn. (15) and "Ramp Filter" stands for the conventional ramp filtering. A NVIDIA Quadro FX 5800 was used for GPU implementation (Cuda 4.0). CPU results were computed in a single thread on an Intel ${ }^{\circledR}$ Xeon X5570.

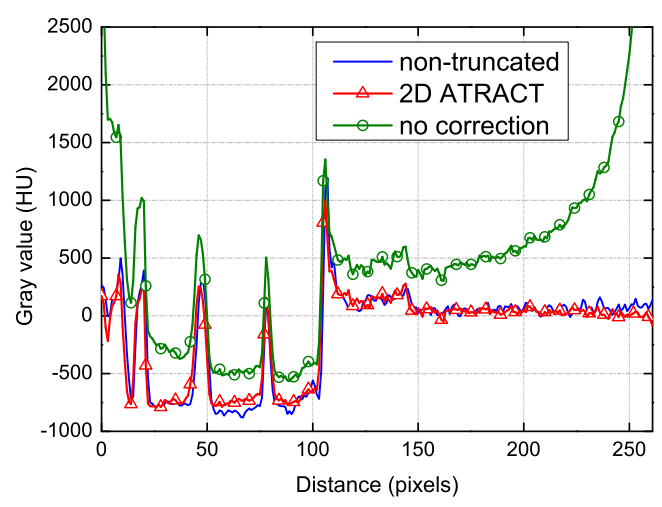

Fig. 8. Profiles along the yellow-dashed line in the clinical case I.

the portions of the patient inside the FOV are almost identical to the ground truth. Further analysis is provided by the central profiles through the transversal slices indicated as the yellowdashed line (see Fig. 8). Note that the differences between the FDK-based full FOV reconstruction and ATRACT-based VOI reconstruction not only rely on the truncation artifacts, but also depend on the level of physical effects, such as X-ray scattering or polychromatic effects in the projections.

The two transversal slices of reconstructions of clinical case II are given in Fig. 7 and the central profiles through the slices indicated as the yellow-dashed lines are given in Fig. 9 and 10. We can see that the new ATRACT method still yields a high quality in terms of truncation correction even in presence of a narrow and off-centered collimation. The ATRACT reconstruction's accuracy is comparable to that of FDK from the non-truncated projections. The line profiles from Fig. 9 and 10 also demonstrate this observation.

\section{CONCLUSION}

In this paper, we developed an efficient truncation correction that uses neither explicit extrapolation nor prior knowledge for cone-beam VOI imaging. This approach is based on a decomposition of the standard ramp filter into a Laplace operation and a convolution-based residual filter. The proposed 

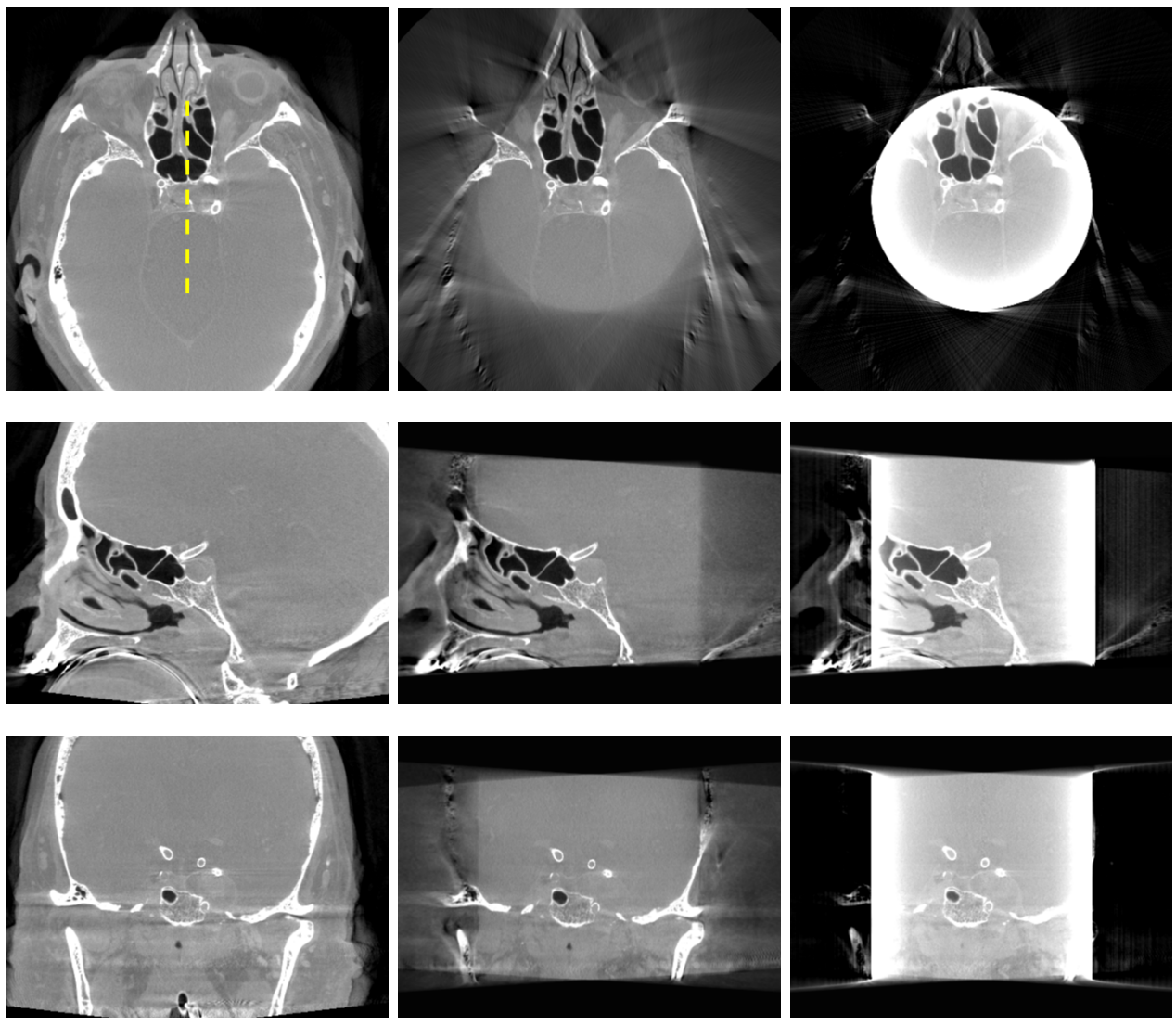

Fig. 6. Reconstruction results of the clinical case I by various algorithms, in the grayscale window [-1000HU, 1000HU]. From left to right: Gold standard reconstruction from non-truncated projection, 2D ATRACT-based VOI reconstruction, FDK-based VOI reconstruction without correction. From top to bottom: transversal view, sagittal view, coronal view.
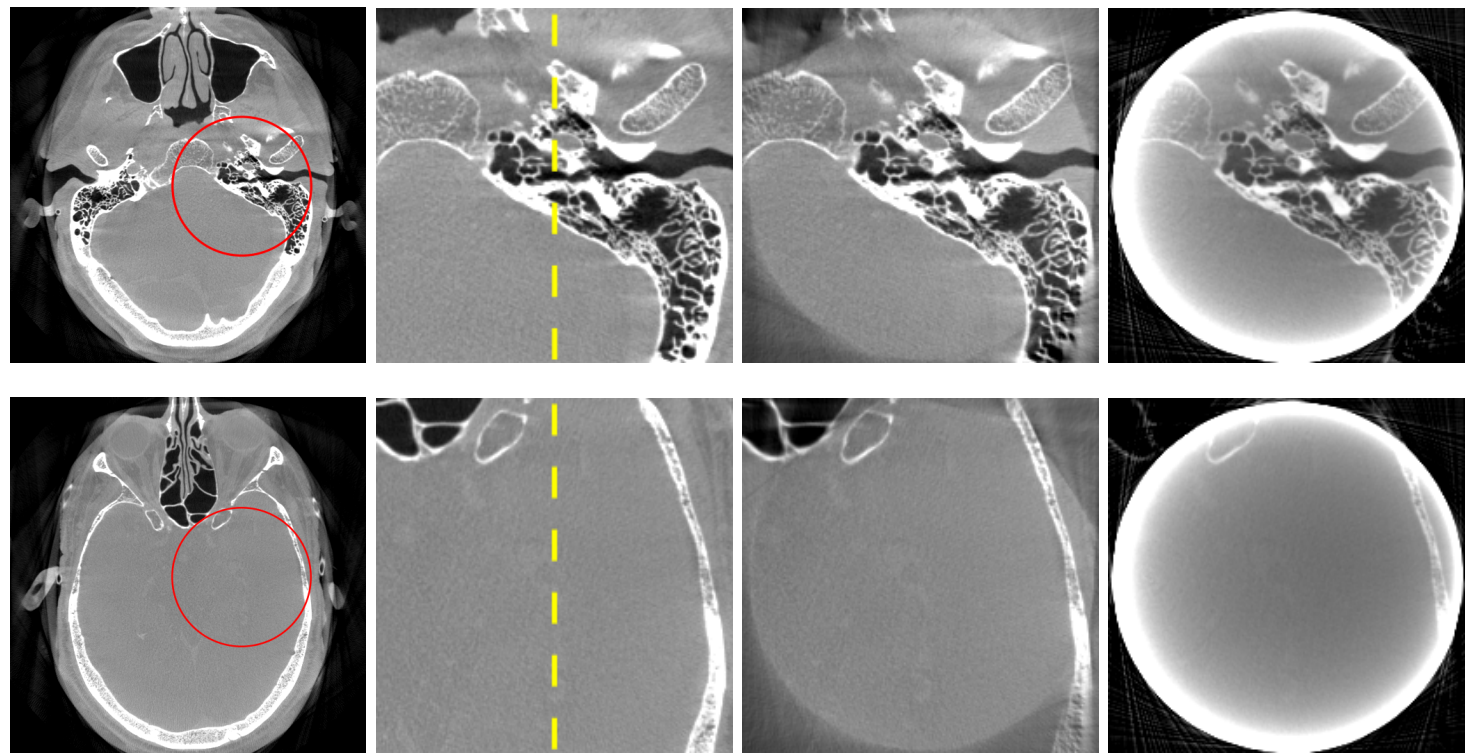

Fig. 7. Two transversal slices of the clinical case II by various algorithms. From left to right: Gold standard reconstruction from non-truncated projection (display window [-1000HU, 1000HU]), zoomed gold standard reconstruction, zoomed 2D ATRACT-based VOI reconstruction, zoomed FDK-based VOI reconstruction without correction (display window $[-1000 \mathrm{HU}, 3000 \mathrm{HU}])$. Red cycles indicate the region of interest. 


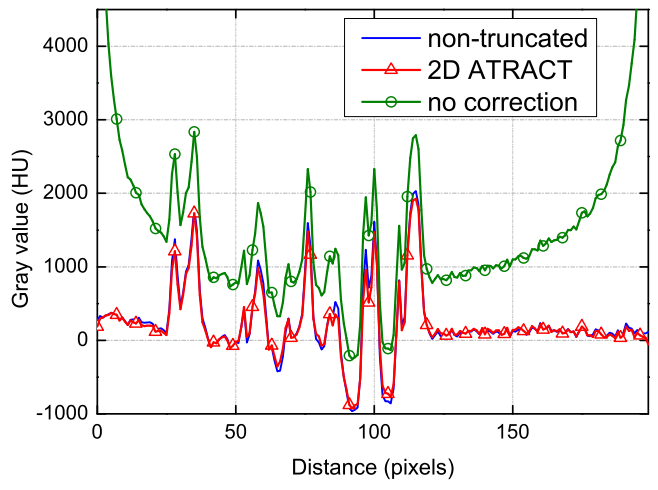

Fig. 9. Profiles along the yellow-dashed line in the clinical case II (top row).

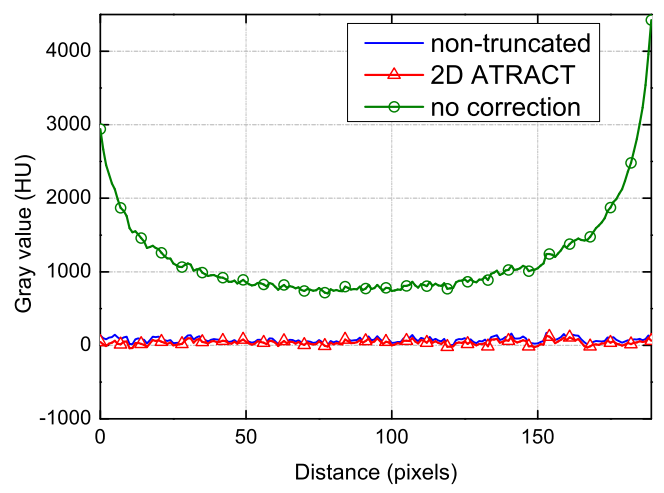

Fig. 10. Profiles along the yellow-dashed line in the clinical case II (bottom row).

algorithms were evaluated by using a reconstruction benchmark test, and two clinical data sets in terms of computational efficiency as well as robustness of correction quality. The new algorithm showed significant improvement on computational efficiency compared to the original one, e.g. a speed-up factor of 860 was achieved due to the efficient 2D filtering and the GPU implementation. This feature ensures new ATRACT be well-suited for interventional CT imaging or image-guided radiation therapy. Furthermore, the clinical evaluation demonstrated that the proposed algorithm maintained the reconstruction of high accuracy even in presence of highly truncated data.

\section{REFERENCES}

[1] F. Noo, R. Clackdoyle, and J. D. Pack, "A Two-step Hilbert Transform Method for 2D Image Reconstruction," Physics in Medicine and Biology, vol. 49, no. 17, pp. 3903-3923, 2004.

[2] X. Pan, Y. Zhou, and D. Xia, "Image reconstruction in peripheral and central region-of-interest and data redundancy," Medical Physics, vol. 32, no. 3, pp. 673-684, 2005.

[3] M. Defrise, F. Noo, R. Clackdoyle, and H. Kudo, "Truncated Hilbert transform and image reconstruction from limited tomographic data," Inverse Problems, vol. 22, no. 3, pp. 1037-1053, 2006.

[4] Y. Ye, H. Yu, and G. Wei, "Exact interior reconstruction with cone-beam ct," International Journal of Biomedical Imaging, vol. 2007, p. 10693, 2007.

[5] — - "Exact interior reconstruction from truncated limited-angle projection data," International Journal of Biomedical Imaging, vol. 2008, p. 427989, 2008.

[6] H. Kudo, M. Courdurier, F. Noo, and M. Defrise, "Tiny a priori knowledge solves the interior problem in computed tomography," Physics in Medicine and Biology, vol. 52, no. 9, p. 2207, 2008.
[7] R. Chityala, K. R. Hoffmann, D. R. Bednarek, and S. Rudin, "Region of Interest (ROI) Computed Tomography," Proceedings of Society PhotoOptical Instrumentation Engineers, vol. 5368, no. 2, pp. 534-541, 2004.

[8] D. Kolditz, Y. Kyriakou, and W. A. Kalender, "Volume-of-interest (VOI) imaging in $\mathrm{C}$-arm flat-detector CT for high image quality at reduced dose," Medical Physics, vol. 37, no. 6, pp. 2719-2730, 2010.

[9] D. Kolditz, M. Meyer, Y. Kyriakou, and W. A. Kalender, "Comparison of extended field-of-view reconstructions in C-arm flat-detector CT using patient size, shape or attenuation information," Physics in Medicine and Biology, vol. 56, no. 1, pp. 39-56, 2011.

[10] B. Ohnesorge, T. Flohr, K. Schwarz, J. P. Heiken, and J. P. Bae, "Efficient correction for CT image artifacts caused by objects extending outside the scan field of view," Medical Physics, vol. 27, no. 1, pp. 39-46, 2000.

[11] J. Hsieh, E. Chao, J. Thibault, B. Grekowicz, A. Horst, S. McOlash, and T. J. Myers, "A novel reconstruction algorithm to extend the CT scan field-of-view," Medical Physics, vol. 31, no. 9, pp. 2385-2391, 2004.

[12] K. Sourbelle, M. Kachelriess, and W. A. Kalender, "Reconstruction from truncated projections in CT using adaptive detruncation," European Radiology, vol. 15, no. 5, pp. 1008-1014, 2005.

[13] J. D. Maltz, S. Bose, H. P. Shukla, and A. R. Bani-Hashemi, "CT truncation artifact removal using water-equivalent thicknesses derived from truncated projection data," in Proc. Annu. Int. Conf. IEEE Engineering in Medicine and Biology Society, 2007, pp. 2905-2911.

[14] A. Maier, B. Scholz, and F. Dennerlein, "Optimization-based Extrapolation for Truncation Correction," in 2nd CT Meeting, 2012, pp. 390-394.

[15] F. Dennerlein, "Cone-beam ROI reconstruction using the Laplace operator," in Proceedings of Fully 3D 2011, 2011, pp. 80-83.

[16] L. A. Feldkamp, L. C. Davis, and J. W. Kress, "Practical cone beam algorithm," Optical Society of America, vol. 1, pp. 612-619, 1984.

[17] F. Dennerlein and A. Maier, "Region-of-interest reconstruction on medical C-arms with the ATRACT algorithm," in Proceedings of SPIE, 2012, p. 83131B.

[18] C. Rohkohl, B. Keck, H. G. Hofmann, and J. Hornegger, "RabbitCTan open platform for benchmarking 3D cone-beam reconstruction algorithms," Medical Physics, vol. 36, no. 9, pp. 3940-3944, 2009. 